\title{
The cost determinants of routine infant immunization services: a meta-regression analysis of six country studies
}

\author{
Nicolas A. Menzies ${ }^{1,2^{*}}$, Christian Suharlim², Fangli Geng ${ }^{2}$, Zachary J. Ward ${ }^{2}$, Logan Brenzel ${ }^{3}$ and Stephen C. Resch ${ }^{2}$
}

\begin{abstract}
Background: Evidence on immunization costs is a critical input for cost-effectiveness analysis and budgeting, and can describe variation in site-level efficiency. The Expanded Program on Immunization Costing and Financing (EPIC) Project represents the largest investigation of immunization delivery costs, collecting empirical data on routine infant immunization in Benin, Ghana, Honduras, Moldova, Uganda, and Zambia.

Methods: We developed a pooled dataset from individual EPIC country studies (316 sites). We regressed log total costs against explanatory variables describing service volume, quality, access, other site characteristics, and income level. We used Bayesian hierarchical regression models to combine data from different countries and account for the multi-stage sample design. We calculated output elasticity as the percentage increase in outputs (service volume) for a $1 \%$ increase in inputs (total costs), averaged across the sample in each country, and reported first differences to describe the impact of other predictors. We estimated average and total cost curves for each country as a function of service volume.
\end{abstract}

Results: Across countries, average costs per dose ranged from $\$ 2.75$ to $\$ 13.63$. Average costs per child receiving diphtheria, tetanus, and pertussis ranged from $\$ 27$ to $\$ 139$. Within countries costs per dose varied widely - on average, sites in the highest quintile were $440 \%$ more expensive than those in the lowest quintile. In each country, higher service volume was strongly associated with lower average costs. A doubling of service volume was associated with a 19\% (95\% interval, 4.0-32) reduction in costs per dose delivered, (range 13\% to 32\% across countries), and the largest $20 \%$ of sites in each country realized costs per dose that were on average $61 \%$ lower than those for the smallest $20 \%$ of sites, controlling for other factors. Other factors associated with higher costs included hospital status, provision of outreach services, share of effort to management, level of staff training/ seniority, distance to vaccine collection, additional days open per week, greater vaccination schedule completion, and per capita gross domestic product.

Conclusions: We identified multiple features of sites and their operating environment that were associated with differences in average unit costs, with service volume being the most influential. These findings can inform efforts to improve the efficiency of service delivery and better understand resource needs.

Keywords: Economic evaluation, Costs and cost analyses, Immunization programs, Vaccination

\footnotetext{
* Correspondence: nmenzies@hsph.harvard.edu

'Department of Global Health and Population, Harvard T.H. Chan School of

Public Health, Boston, Massachusetts, USA

${ }^{2}$ Center for Health Decision Science, Harvard T.H. Chan School of Public

Health, Boston, Massachusetts, USA

Full list of author information is available at the end of the article
} 


\section{Background}

Better evidence on immunization costs serves several goals - in the context of new vaccine adoption and service expansion, accurate cost estimates are critical inputs for cost-effectiveness and budget impact analyses of proposed policies. Understanding variation in costs across service outlets can also provide insight on site operations and suggest opportunities for improving efficiency. Despite these needs, there have been few empirical studies with sufficient sample size to provide precise cost estimates or describe inter-site cost variation, and studies have commonly used national-level budgeting data to investigate determinants and trends in immunization costs [1-3]. Prior empirical studies have found substantial variation both within $[4,5]$ and between countries [6], though some of these studies are now many years old.

The Expanded Program on Immunization Costing and Financing (EPIC) Project was designed to fill this knowledge gap, providing detailed data on routine immunization costs and financing in a large, representative sample of immunization sites in six countries (Benin, Ghana, Honduras, Moldova, Uganda, and Zambia) [7]. Information from these studies has already been used to improve information on unit costs [8-11], cost trends [12,13], and financing $[14,15]$ within individual countries. We synthesized data from these country studies to create a unique pooled dataset of 316 sites to explore cross-country determinants of costs. We use these data to describe countryand site-level variation in routine immunization costs and identify systematic cost differences related to site operating characteristics. Given the observational nature of the data, the relationships we estimate are purely descriptive. However, the larger sample size allows us to provide a finegrained description of how costs vary between similar sites, which in turn can suggest potential approaches for improving the efficiency of service delivery and allow a more precise understanding of resource needs.

\section{Methods}

\section{Study sample}

Countries were selected from a list of countries that had introduced pentavalent vaccine before 2011, and introduced pneumococcal or rotavirus vaccines in 2011 [7]. Countries varied by program performance (diphtheria, tetanus, and pertussis (DTP3) coverage from $75 \%$ to $93 \%$ [16]), income level (gross domestic product (GDP) per capita from US \$531 to US \$2277 [17]), and vaccination schedule. Inter-country differences are described in Additional file 1: Table S1. Sites were selected using a multi-stage cluster sample from a sampling frame of all public and non-governmental organization (NGO) facilities providing routine immunization. A total of 319 sites were enrolled. From each site, data were collected on costs, service volume, and site operating characteristics during January-December 2011, using a common approach [18]. Data were also collected on costs incurred at the subnational and national levels. Data collection occurred during 2012-2013.

\section{Data management and cleaning}

We collated detailed data files from individual country studies and compiled them into a consistent format, with costs organized into standardized cost categories. We used automated tests to identify data anomalies, which were discussed and resolved with country teams. Three sites were excluded due to unresolved inconsistencies. Data cleaning and standardization resulted in minor differences with earlier studies' country-level results [8-11]. Datasets and related materials are available at www.immunizationeconomics.org and archived on Dataverse [19].

\section{Total cost estimates}

Total site-level economic costs were estimated from a program perspective using conventional methods, including all activities undertaken to provide routine immunization services to children aged 0-12 months. We focused on the 0-12 month old age group to allow greater standardization across the data collected through individual country-level studies. Site-level overheads were apportioned by direct allocation [18], and investments were amortized with a $3 \%$ discount rate [20]. Building space was costed as equivalent rental value or annualized construction cost, and donated items valued at market prices. Costs for equipment and other investments were based on replacement value. Costs for salaried labor were based on loaded salaries, and costs for non-salaried labor ("volunteers") included volunteer stipends, per diems, and incentives. Additional file 1: Table S2 provides a full inventory of costs included in the study. Costs are reported in 2011 US dollars.

\section{Service delivery volume}

Data on service delivery volume were extracted from routine reporting systems. We used the number of total immunization doses delivered to children aged 0-12 months by each site during the study period as the primary measure of service volume. We also report results using the number of children receiving their third immunization for DTP3 by each site during the study period as an alternate measure of service volume. DTP3 is a conventional proxy for the number of children completing the basic infant immunization schedule.

\section{Average cost estimates}

For this study, we estimated the average cost per site, per dose, and per DTP3 for each country, and obtained confidence intervals with a multi-stage bootstrap with resampling at province and site levels. Average costs per 
dose and DTP3 were calculated as the sum of routine infant immunization costs for all sites in the sample divided by the sum of outputs (doses, or DTP3) for all sites in the sample. This value is equivalent to a weighted average of the site-level cost per outcome, with weights proportional to service volume. This represents an estimate of the cost per outcome assessed at the program level. We also report results as a simple average of the cost per outcome for all sites in the sample. Average cost estimates are reported with and without higher level program support ("above site costs") included. All estimates were adjusted for survey weighting, with weights constructed as the inverse of the sampling probability for each site [18].

\section{Regression analyses}

We explored site-level cost variation by regressing the log of total costs incurred at each site against several explanatory variables. We excluded higher level program support (above site costs) from this part of the analysis, as these costs are unlikely be explained by the site-level determinants considered as explanatory variables. We used a Bayesian hierarchical regression model to combine data from different country studies and account for the multistage sample design, with country- and province-level random effects. Using a hierarchical regression model allowed for sources of variation at the site, province, and country levels, and provided a framework for synthesizing data across countries. As we used a Bayesian approach, the uncertainty measures included in the Results section (such as $95 \%$ credible intervals provided around point estimates) represent posterior probabilities conditional on priors, likelihood, and regression model, unlike traditional confidence intervals. Regression models were estimated using an adaptive Hamiltonian Monte Carlo algorithm [21, 22]. Processing of data and results were undertaken in $\mathrm{R}$ [23]. Further details are provided in Additional file 1: Framework for regression analyses.

Explanatory variables included service volume (log(doses)), other site characteristics (government ownership (Govt owned), hospital status (Hospital) as an indicator of health system level, fraction of DTP3 delivered via outreach (Fraction outreach), fraction of resources devoted to management (Fraction mgmt), and the ratio of DTP3 to doses (DTP3 per dose) as a crude quality measure), and features of the operating environment (rural location (Rural), local antenatal care (ANC) coverage as a measure of healthcare access (ANC4), and wealth level in the local area relative to the national average (Wealth ratio)). We also included log per capita GDP in $2011(\log (G D P))$ as a crude index of inter-country price differences and other factors that vary with income level.
We fit a series of progressively more inclusive regression models including these explanatory variables. Model 1 involves intercept plus country- and provincelevel random effects. Model 2 incorporates Model 1 plus $\log ($ doses $)$ and $\log$ (doses) squared. Model 3 incorporates Model 2 plus $\log (\mathrm{GDP})$ and site characteristics. Model 4 incorporates Model 3 plus features of the operating environment. Model 5 incorporates Model 4 plus country-level random effects for $\log$ (doses).

Several variables were unavailable for some sites, and we investigated these in secondary analyses using the subset of sites for which data were available. As salary scales were fixed within each country, we used the site-level average salary, normalized to 1.0 for each country, as an index of staff training and seniority (Staffing index, not available for 10 sites). We also created an index for the average fraction of time staff spent working on immunization (Dedication index, not available for Uganda) to describe the extent to which staff were committed exclusively to immunization activities, as compared to being spread across multiple service areas. This was used as a measure of economies of scope for labor. Since the definition of a hospital may differ across countries, we used the number of inpatient beds, categorized into 0 beds, $1-9$ beds, or 10 + beds, as an alternative indicator of health system level (Inpatient beds, not available for Moldova). Finally, we considered the number of days open per week (Days per week) and the distance to the vaccine collection point (Distance), both not available for Honduras. Table 1 provides summary information on all predictors. Additional file 1: Table S3 provides detailed variable definitions.

We estimated a series of progressively more inclusive regression models, using the Watanabe-Akaike information criterion (WAIC) to describe model fit [24, 25]. Similar to AIC, WAIC is a statistic that measures the extent to which a model is able to explain variation in the data, while penalizing unnecessary model complexity. A lower WAIC implies a better fitting model. We calculated first differences using the regression results, describing the change in the cost per dose associated with a defined change in one or several predictors. We present equaltailed $95 \%$ credible intervals to describe the uncertainty in these results, and we use the term "statistically discernable" to describe situations where these intervals exclude no effect. We generated graphs of total and average costs per dose as a function of service volume, using the Duan smearing estimator to retransform estimates to the absolute scale [26]. We calculated output elasticity as the percentage increase in outputs (service volume) for a $1 \%$ increase in inputs (total costs), averaged across the sample in each country. Using the fitted regression equations, we compared predicted unit costs (total costs divided by total doses) across the range of service delivery volume observed in each country, controlling for other factors. 
Table 1 Characteristics of sample

\begin{tabular}{|c|c|c|c|c|c|c|}
\hline Outcome & Benin & Ghana & Honduras & Moldova & Uganda & Zambia \\
\hline Sample size ${ }^{a}$ & 45 & 50 & 71 & 50 & 49 & 51 \\
\hline Total doses & $7014(4684)$ & $3512(3775)$ & $4244(7175)$ & $557(1172)$ & $6561(12144)$ & 7069 (11343) \\
\hline Total DTP3 & $665(465)$ & $378(358)$ & $280(421)$ & $54(111)$ & $682(1401)$ & $708(1006)$ \\
\hline Per capita GDP, 2011 USD & $\$ 745$ & $\$ 1594$ & $\$ 2277$ & $\$ 1971$ & $\$ 531$ & $\$ 1741$ \\
\hline Rural & $25 / 45$ & $31 / 50$ & $53 / 71$ & $42 / 50$ & $29 / 49$ & $36 / 51$ \\
\hline Government owned & $41 / 45$ & $47 / 50$ & $71 / 71$ & $50 / 50$ & $37 / 49$ & $49 / 51$ \\
\hline Hospital & $0 / 45$ & $6 / 50$ & $3 / 71$ & $0 / 50$ & $13 / 49$ & $4 / 51$ \\
\hline ANC4 & $0.69(0.13)$ & $0.83(0.04)$ & $0.90(0.03)$ & $0.93(0.01)$ & $0.52(0.06)$ & $0.62(0.02)$ \\
\hline Wealth ratio & $1.17(0.31)$ & $1.03(0.26)$ & $1.09(0.36)$ & $0.92(0.30)$ & $1.10(0.47)$ & $1.42(0.45)$ \\
\hline Fraction outreach & $0.20(0.21)$ & $0.63(0.32)$ & $0.14(0.07)$ & $0.00(0.00)$ & $0.38(0.08)$ & $0.46(0.20)$ \\
\hline Fraction management & $0.02(0.03)$ & $0.07(0.06)$ & $0.05(0.05)$ & $0.19(0.06)$ & $0.13(0.06)$ & $0.12(0.07)$ \\
\hline Staffing index & $1.00(0.54)$ & $1.00(0.15)$ & $1.00(0.35)$ & $1.00(0.25)$ & $1.00(0.28)$ & $1.00(0.32)$ \\
\hline DTP3 per dose & $0.10(0.03)$ & $0.12(0.06)$ & $0.08(0.01)$ & $0.10(0.02)$ & $0.11(0.02)$ & $0.11(0.02)$ \\
\hline Days open per week & $3.8(1.5)$ & $4.3(1.9)$ & - & $3.9(1.6)$ & $2.7(2.2)$ & $1.9(1.5)$ \\
\hline Distance to vaccine collection point $(\mathrm{km})$ & $15.5(18.5)$ & $8.0(11.5)$ & - & $19.6(13.1)$ & $12.9(12.6)$ & $50.2(44.8)$ \\
\hline Dedication index & $0.50(0.27)$ & $0.45(0.22)$ & $0.34(0.15)$ & $0.26(0.11)$ & - & $0.32(0.19)$ \\
\hline Inpatient beds: 0 & $0 / 45$ & $11 / 50$ & $68 / 68$ & - & $10 / 49$ & $12 / 50$ \\
\hline Inpatient beds: 1-9 & $12 / 45$ & $34 / 50$ & $0 / 68$ & - & $17 / 49$ & $22 / 50$ \\
\hline Inpatient beds: $10+$ & $33 / 45$ & $5 / 50$ & $0 / 68$ & - & $22 / 49$ & $16 / 50$ \\
\hline Total catchment population (000 s) & $20.7(17.3)$ & $14.1(21.8)$ & - & $5.2(11.1)$ & $41.4(98.5)$ & $22.2(37.6)$ \\
\hline DTP3 coverage ${ }^{\mathrm{b}}$ & $0.82(0.25)$ & $0.75(0.26)$ & $0.85(0.18)$ & $0.88(0.16)$ & $0.62(0.35)$ & $0.81(0.21)$ \\
\hline
\end{tabular}

${ }^{a}$ Sample size values represent the number of sites included in the main analysis for each country. All other values in table represent unweighted means for each county, and values in parentheses represent standard deviations

${ }^{\mathrm{b}}$ Top-coded at $100 \%$

\section{Robustness checks}

We estimated several alternative regression specifications: (1) country-level fixed effects instead of random effects; (2) addition of district-level random effects; (3) a robust regression specification, with residuals assumed to follow a Student's $t$ distribution [27], to allow for outliers; and (4) adoption of non-informative priors (instead of weakly informative priors) for regression coefficients and variance terms. We also estimated results using the number of children receiving DTP3 as a measure of service volume in place of total doses.

We fit several regression models to investigate coverage as a predictor of site-level costs. We anticipated that unit costs of service delivery would be increasing at higher coverage levels, due to high marginal costs of reaching the very last members of the target population. However, the reported coverage measure (DTP3 coverage, reported as DTP3 divided by number of children $<1$ years old in the catchment) exhibited substantial measurement error (many values $>100 \%$ ), attributed to inaccurate population estimates. We omitted DTP3 coverage from the main analysis but undertook sensitivity analyses with (1) reported DTP3 coverage top-coded at $100 \%$ (i.e., revised to a value of $100 \%$ where the original value was >100\%), (2) an error-in-variables model for mismeasurement of DTP3 coverage, and (3) use of log catchment population $\left(\log \left(\mathrm{Po}_{\mathrm{O}}\right.\right.$ pulation)) as an indirect approach for investigating the relationship between coverage and costs.

\section{Results}

\section{Average cost estimates}

In 2011 the study sites spent $\$ 6.79$ million (2011 US dollars (USD)) providing routine immunization services to children $<1$ year of age, recording 1.50 million vaccine doses and 141,000 DTP3. The distributions of sitelevel costs and service volume were strongly right skewed, with many small sites and few large sites. There was substantial variation in the cost per dose and per DTP3 in each country, with the coefficient of variation in the cost per dose ranging from 0.45 in Moldova to 1.37 in Ghana (mean 0.99 across the entire sample), such that the quintile of sites with the highest cost per dose were on average $440 \%$ more expensive than the quintile with the lowest cost per dose in each country. Additional file 1: Figure S1 shows distributions of the cost per site, per dose, and per DTP3 by country.

Table 2 reports average costs per site, per dose, and per DTP3 we estimated for each country. Average costs 


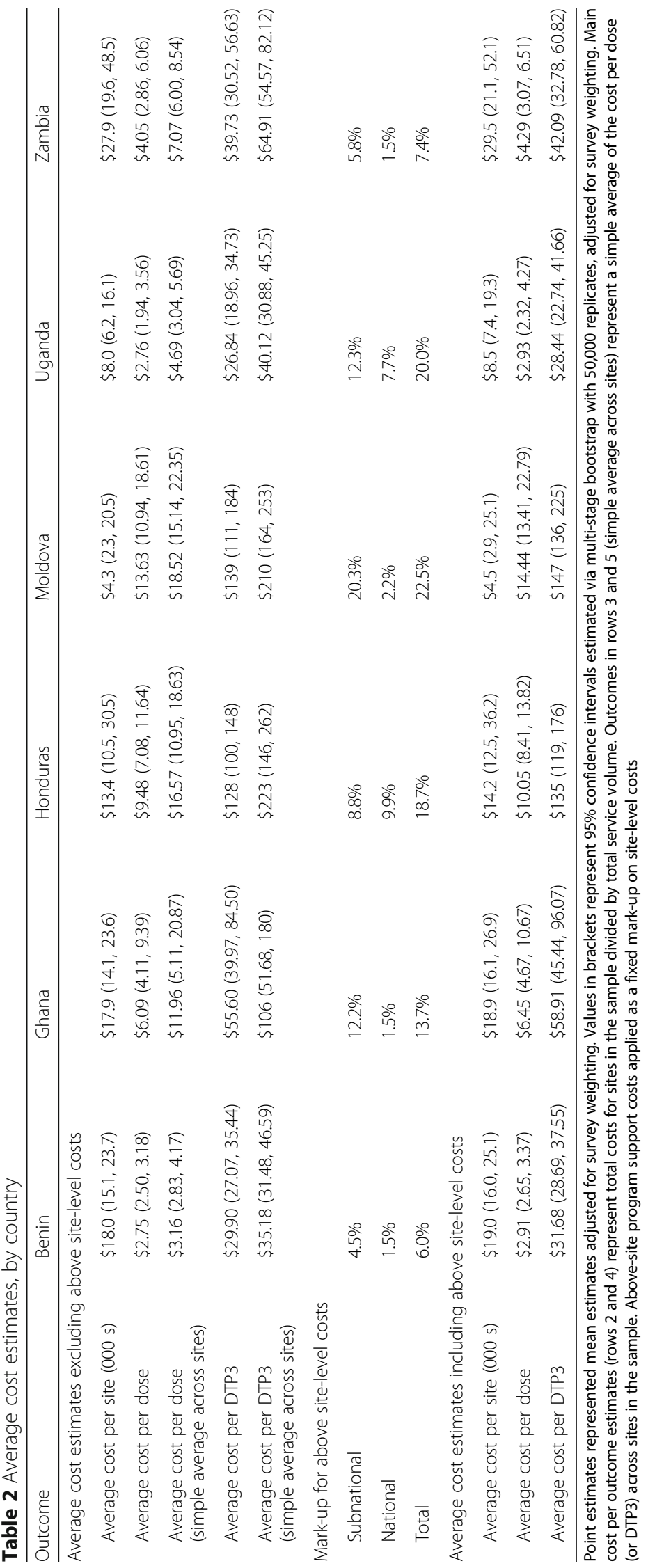


per site ranged from $\$ 4300$ in Moldova to $\$ 27,900$ in Zambia. Average costs per dose ranged from $\$ 2.75$ in Benin to $\$ 13.63$ in Moldova. Average costs per DTP3 ranged from \$26.84 in Uganda to \$139 in Moldova. Cost per outcome estimates calculated as a simple average across sites were $15-96 \%$ higher than values weighted by service volume, implying that sites with higher service volume had lower costs per outcome. Overheads for costs incurred above the site level represented an additional $6.0-22.5 \%$ on top of site-level costs.

\section{Cost determinants}

Table 3 reports a series of progressively more inclusive regression models fit to log total costs.

Results show a strong relationship between service volume and total costs, evident in the substantial reduction in WAIC produced by including service volume in the regression model. Including variables for site characteristics and features of the operating environment produced additional reductions in WAIC (though insignificant in the latter case). The additional reduction in WAIC associated with inclusion of random effects for $\log$ (doses) (Model 5 vs. Model 4) indicates statistically discernable inter-country variation in the relationship between total costs and service delivery volume.

As the regressions used log-transformed costs and standardized variables, the regression coefficients are difficult to interpret directly [28]. First differences were calculated to demonstrate the implications of the regression results (Table 4), based on the best fitting regression model (Model 5). These results describe the percentage difference in the cost per dose produced by change in an individual predictor or subset of predictors. As the regression model included service volume, the first differences

Table 3 Results for regressions of log total cost on service volume and other potential predictors

\begin{tabular}{|c|c|c|c|c|c|}
\hline \multirow[t]{2}{*}{ Variable $^{a}$} & \multicolumn{5}{|c|}{ Model specification } \\
\hline & 1 & 2 & 3 & 4 & 5 \\
\hline Intercept & $9.44(0.45)$ & $9.44(0.25)$ & $9.43(0.16)$ & $9.43(0.16)$ & $9.48(0.13)$ \\
\hline \multicolumn{6}{|l|}{ Service volume } \\
\hline $\log ($ doses $)$ & - & $1.10(0.03)$ & $1.10(0.03)$ & $1.13(0.04)$ & $1.04(0.20)$ \\
\hline $\log ($ doses $)$ squared & - & $-0.04(0.02)$ & $-0.01(0.02)$ & $-0.01(0.02)$ & $0.10(0.03)$ \\
\hline \multicolumn{6}{|l|}{ Other predictors } \\
\hline $\log (G D P)$ & - & - & $0.41(0.16)$ & $0.31(0.17)$ & $0.39(0.14)$ \\
\hline Government owned & - & - & $-0.13(0.09)$ & $-0.14(0.09)$ & $-0.15(0.08)$ \\
\hline Hospital & - & - & $0.34(0.09)$ & $0.31(0.09)$ & $0.27(0.08)$ \\
\hline Percent outreach & - & - & $0.11(0.04)$ & $0.11(0.04)$ & $0.08(0.03)$ \\
\hline Percent management & - & - & $0.13(0.03)$ & $0.13(0.03)$ & $0.13(0.03)$ \\
\hline DTP3 per dose & - & - & $0.15(0.02)$ & $0.15(0.02)$ & $0.13(0.02)$ \\
\hline Rural & - & - & - & $-0.02(0.06)$ & $-0.04(0.06)$ \\
\hline ANC4 & - & - & - & $0.14(0.08)$ & $0.12(0.07)$ \\
\hline Wealth ratio & - & - & - & $-0.06(0.04)$ & $-0.07(0.03)$ \\
\hline \multicolumn{6}{|l|}{ Random effects included } \\
\hline Country r.e.s for intercept & + & + & + & + & + \\
\hline Province r.e.s for intercept & + & + & + & + & + \\
\hline Country r.e.s for log(doses) & - & - & - & - & + \\
\hline \multicolumn{6}{|l|}{ Variance parameters } \\
\hline Error term & $0.86(0.04)$ & $0.39(0.02)$ & $0.35(0.02)$ & $0.35(0.02)$ & $0.32(0.01)$ \\
\hline SD of country r.e.s, intercept & $0.93(0.48)$ & $0.55(0.27)$ & $0.34(0.24)$ & $0.32(0.23)$ & $0.24(0.21)$ \\
\hline SD of province r.e.s, intercept & $0.44(0.10)$ & $0.19(0.04)$ & $0.19(0.04)$ & $0.20(0.04)$ & $0.14(0.03)$ \\
\hline SD of country r.e.s, log(doses) & - & - & - & - & $0.46(0.23)$ \\
\hline WAIC $^{\mathrm{b}}$ & 828.2 & 335.2 & 273.2 & 270.9 & 212.4 \\
\hline Sample size & 316 & 316 & 316 & 316 & 316 \\
\hline
\end{tabular}

${ }^{a}$ Country and province random effects not shown. Predictors are standardized; thus, fitted coefficients for continuous variables (e.g., log(doses)) represent the increase in log total costs observed for a 1.0 standard deviation increase in the variable. Values in parentheses represent standard errors

${ }^{b}$ Watanabe-Akaike information criterion (WAIC) describes out-of-sample prediction accuracy for the fitted model, with lower values suggesting better model fit $S D$ standard deviation, r.e. random effect 
Table 4 First differences calculated from regression results

\begin{tabular}{|c|c|}
\hline Comparison $^{a}$ & Percentage difference in average cost per dose \\
\hline \multicolumn{2}{|c|}{ Each country, as compared to the overall mean (includes differences in per-capita GDP, controls for other predictors): } \\
\hline Uganda (per capita GDP 2011 = US\$ 531) & $-55 \%(-65,-42)$ \\
\hline Benin (per capita GDP $2011=$ US\$ 745) & $-27 \%(-44,-8.2)$ \\
\hline Ghana (per capita GDP 2011 = US\$ 1594) & $-9.6 \%(-26,8.1)$ \\
\hline Zambia (per capita GDP $2011=$ US\$ 1740) & $22 \%(1.4,47)$ \\
\hline Moldova (per capita GDP 2011 = US\$ 1971) & $27 \%(2.8,53)$ \\
\hline Honduras (per capita GDP $2011=$ US\$ 2277) & $43 \%(21,65)$ \\
\hline Per capita GDP doubled & $68 \%(16,133)$ \\
\hline Government-owned sites, as compared to non-government-owned sites & $-14 \%(-27,-0.8)$ \\
\hline Hospital-based sites, as compared to other sites & $32 \%(12,55)$ \\
\hline Percentage of doses delivered via outreach $32 \%$ points higher ${ }^{c}$ & $9.8(1.6,18)$ \\
\hline Share of program activity to management $12 \%$ points higher $^{c}$ & $23 \%(12,33)$ \\
\hline DTP3 as percent of all doses $1.5 \%$ points higher ${ }^{c}$ & $13 \%(8.4,18)$ \\
\hline Rural sites, as compared to urban and per-urban sites & $-3.6 \%(-14,7.5)$ \\
\hline ANC4 coverage $30 \%$ points higher ${ }^{c}$ & $25 \%(-2.5,61)$ \\
\hline Wealth ratio $52 \%$ points higher ${ }^{c}$ & $-8.8 \%(-16,-0.8)$ \\
\hline \multicolumn{2}{|c|}{ Service delivery volume (doses) doubled, as compared to a site with the median no. doses for each country: } \\
\hline Uganda & $-14 \%(-19,-7.7)$ \\
\hline Benin & $-16 \%(-23,-7.9)$ \\
\hline Ghana & $-32 \%(-37,-27)$ \\
\hline Zambia & $-27 \%(-32,-22)$ \\
\hline Moldova & $-13 \%(-17,-8.9)$ \\
\hline Honduras & $-18 \%(-21,-14)$ \\
\hline Overall & $-19 \%(-32,-4.0)$ \\
\hline
\end{tabular}

${ }^{a}$ Values calculated from the results of regression Model 5, controlling for all other model parameters except for those described in the comparison

bCalculated as one minus the average cost per dose for the given scenario divided by average cost per dose in comparator scenario (thus, "-50\%" would indicate

a halving of costs and " $50 \%$ " would indicate a $50 \%$ increase in costs). Values represent posterior means, and values in parentheses represent equal-tailed $95 \%$ credible intervals

${ }^{c}$ Magnitude of change equal to the difference between the 25 th and the 75 th percentile of the sample distribution for each variable

calculated for other predictors apply to both the cost per dose and total site-level costs, for sites of equal service volume. For continuous predictors, first differences were calculated to represent the difference between the $25 \%$ and $75 \%$ percentiles of each predictor.

Figure 1 presents total and average costs for each country as a function of service volume, based on Model 5. Additional file 1: Figure S2 presents these relationships plotted on a log scale to allow better visualization of model fit. The average cost curves reveal differences between countries, though all curves reflect a convex, monotonically declining relationship between average costs and service volume. These results demonstrate a strong negative relationship between service volume and average costs in each country, and we estimated output elasticity values of 1.39 (1.16-1.68), 2.52 (1.97-3.31), 1.41 (1.31-1.54), 1.27 (1.17-1.40), 1.34 (1.19-1.52), and 1.87 (1.56-2.28) in Benin, Ghana, Honduras, Moldova,
Uganda, and Zambia respectively. This implies that, across the entire sample, a site with $10 \%$ higher costs would have on average 14\% (12-17) higher service volume, controlling for other effects. As a consequence, the largest $20 \%$ of sites (by number of doses) were estimated to have a cost per dose that was 55\% (40-67), $41 \%$ (24-55), 83\% (77-88), 68\% (59-76), 52\% (38-63), and $67 \%(59-75)$ percent lower than that for the smallest $20 \%$ of sites in Benin, Ghana, Honduras, Moldova, Uganda, and Zambia respectively, controlling for other factors.

\section{Additional predictors}

Several predictors (staffing index, immunization days per week, distance to vaccine delivery, dedication index, and number of inpatient beds) were not available for the entire sample. We estimated regression models for total sitelevel costs using the subset of sites with data available on 


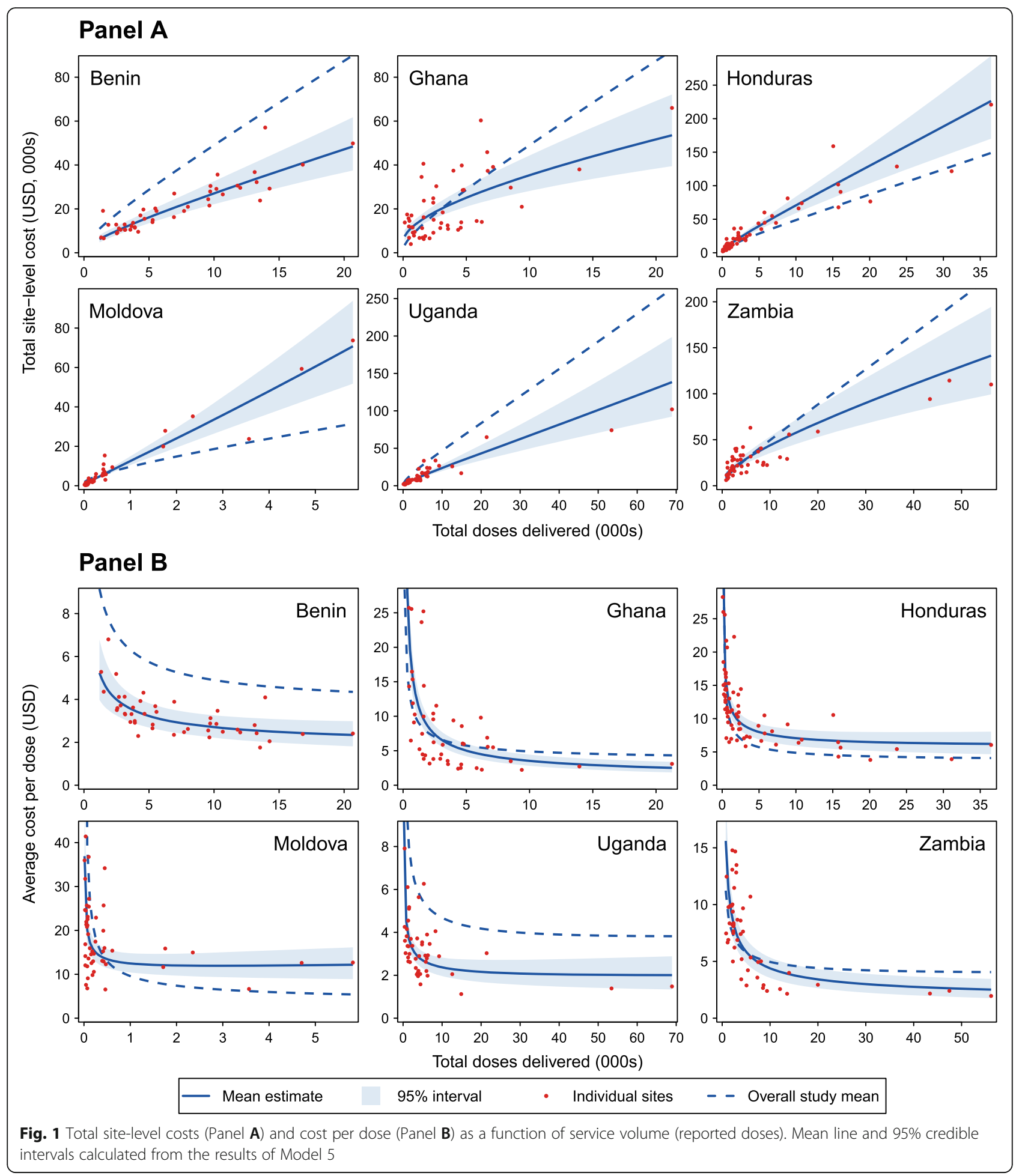

these predictors, and in each case identified a statistically discernable positive relationship with costs (Models 6-10, Additional file 1: Table S4). Coefficients for other predictors showed some variation across these specifications, likely due to the different samples to which models were fit. First differences calculated from these results showed a
5.9\% (95\% credible interval 2.4-9.5) cost increase associated with an 86 percentage point increase in the staffing index (missing for 10 sites). There was a 4.2\% (1.5-7.0) cost increase with each doubling in distance to the vaccine collection point, and a $10.0 \%(2.8-18.0)$ cost increase with each additional day open per week (both variables not 
reported for Honduras). A 25 percentage point increase in the dedication index (extent to which staff were dedicated exclusively to providing immunization services) was associated with a $21 \%(15-28)$ cost increase (excludes Uganda). Finally, when the number of inpatient beds was used as an indicator for health system level (excluding Moldova), sites with $0-9$ beds were 4.7\% ( -9.6 to 19) more expensive than sites without beds, and sites with $10+$ inpatient beds were $21 \%$ (2.3-42) more expensive, consistent with the results for hospital status reported in Table 4.

\section{Alternative regression specifications}

Using country fixed effects instead of random effects did not affect results, with model fit statistics, coefficients, and standard errors universally similar (Additional file 1: Table S5), and a Hausman test failed to reject the random effects specification. Similarly, including district-level random effects (Additional file 1: Table S6), adopting a robust regression approach (Additional file 1: Table S7), or adopting non-informative priors (Additional file 1: Table S8) had little impact on regression results. Additional file 1: Table S9 shows results for regressions using $\log$ (DTP3) as a measure of service volume. This produced little change in most coefficients, except for a minor increase in the strength of the relationship estimated for hospital status, and reversal of the sign of the coefficient for DTP3 per dose. Additional file 1: Figure S3 shows total and average cost curves plotted for DTP3.

Additional file 1: Table S10 presents various approaches for including DTP3 coverage in regression equations. When a top-coded estimate of DTP3 coverage was included, this produced a statistically discernable negative relationship with total costs. Results were similar when an error-in-variables model was used for DTP3 coverage, although no longer statistically discernable. Including log catchment population in the regression equations produced a statistically discernable positive relationship. This is consistent with the two results for DTP3 coverage, as coverage will be negatively related to total catchment population, conditional on service volume.

\section{Discussion}

Costs per dose ranged from $\$ 2.75$ (2011 USD) in Benin and Uganda to $\$ 13.63$ in Moldova. Costs per DTP3 ranged from \$27 in Uganda to \$139 in Moldova. These values are substantially greater than those reported by earlier studies [4-6, 29], reflecting both higher price levels and expanded vaccine schedules. Inter-country differences in this study are likely due to similar factors, with countries with higher costs per DTP3-Moldova and Honduras-experiencing comparatively higher income levels and longer vaccine schedules. Some of our estimates differ from earlier estimates from the EPIC country studies [7], reflecting refinements to cost and outcomes data during data cleaning. Different approaches to calculating the average cost per outcome (Table 2) also contributed to variation in estimates, due to the strong relationship between service volume and the site-level cost per outcome.

Regression analyses revealed several predictors with a statistically discernable relationship with costs. In each country, higher service volume was strongly associated with lower average costs, with output elasticity ranging from 1.27 to 2.52 across the six countries, with a mean value of 1.63 , and the largest $20 \%$ of sites in each country had a cost per dose that was on average $61 \%$ lower than that observed for the smallest $20 \%$ of sites. For most countries there were many small sites with high average costs, and these small sites exhibited substantial variation in unit costs. The reduction in unit costs associated with higher service volume was only minimal for sites at the upper end of the service volume distribution, and these large sites exhibited only minor variation in unit costs. While this suggests that greater reductions in average costs might be achieved through efforts to improve efficiency in small sites, it is not clear that these sites should be prioritized, as large total cost reductions might be possible with only small reductions in unit costs at large sites.

Higher health system level (proxied by hospital status) was associated with higher costs, possibly reflecting differences in available staffing and infrastructure. Outreachbased delivery was also associated with higher costs, possibly due to the additional resources required to deliver mobile services while maintaining infrastructure for facility-based provision. We designed the staffing index to test whether additional productivity of more highly trained and senior staff would counterbalance their higher salaries, yet the positive coefficient on this variable suggests otherwise. Greater distance to vaccine collection and more days open per week (implying lower daily service volume) were both associated with higher costs. A higher fraction of costs for management and greater completion of the vaccination schedule (proxied by the DTP3:doses ratio) were both found to have a strong positive relationship with costs. The positive coefficient on the dedication index implied lower cost for sites where staff are spread across multiple services (rather than being fully committed to immunization services), consistent with economies of scope and a greater ability to adjust staffing with variable demand. Some of these relationships were stronger than others, but they were generally robust to changes in regression specification. After controlling for site characteristics, there was little difference between rural and non-rural sites, despite rural sites having substantially higher costs per dose in crude comparisons. Per capita GDP was found to have a positive relationship with costs per dose. This likely reflects differences in price levels between 
countries, but it could also be related to the many other factors that vary with country income level. As only six countries were represented in this sample, we were unable to decompose the effect of these country-level factors.

The negative coefficient estimated for coverage was unexpected. We expected costs to increase with coverage, reasoning that improving coverage would require attracting progressively harder-to-reach clients, driving up marginal costs. It is possible that for a given service volume, sites with lower coverage have more widely dispersed or immunization resistant catchment populations, leading to higher average costs. Alternately, if staffing and other immunization resources were determined by catchment population size, lower attendance could produce both lower coverage and higher costs per outcome. While mismeasurement of reported coverage dictates that no firm conclusions should be drawn, the importance of coverage for immunization program strategy suggests that further investigation would be valuable.

Several relationships described above have been investigated in country-level studies [12, 13], with generally consistent results. While we have described possible mechanisms for these findings, the ability of our study design to identify causal relationships is weak. Many of the findings are consistent with multiple explanations (including omitted variable bias) and should be viewed as hypothesis generating rather than confirming any specific causal relationship. For this reason, the total and average cost curves we estimate for each country (Fig. 1) demonstrate how costs vary between sites of different size, but additional (and potentially unwarranted) assumptions are required to interpret them as the cost curves that would be experienced by sites attempting to increase service volume. Other limitations include the exclusion of predictors that could not be calculated for a sufficient number of sites to be considered in the analysis (for example, vaccine stock-outs and wastage rates) or that primarily varied at the country level (for example, input prices and vaccine schedules), and for which we had minimal power to investigate. While we attempted to create explanatory variables with consistent interpretations, it is likely that some of these constructs varied between countries, such as the definition of outreach services, or hospital status. Finally, the results for Ghana showed noticeably greater unexplained variation compared to the other countries (Fig. 1, Additional file 1: Figure S2), yet the reasons for this are unclear.

\section{Conclusions}

The EPIC Project is the largest study to systematically investigate routine immunization costs. Prior studies of immunization costs have been hampered by small sample sizes, producing noisy average cost estimates and providing little systematic evidence on the variation in costs within countries. By enrolling a large number of immunization sites and using standardized data collection approaches across countries, the EPIC Project provides a unique opportunity to investigate and understand variation in the costs of immunization service delivery as well as generate more precise national cost estimates. This analysis examined many features of sites and their operating environments, but further investigation of these data is possible. The combined EPIC data are freely available for use by other investigators [19], with the hope that open access will maximize the utility of these data for understanding the costs of routine immunization services. The major findings of this study have relevance for policy makers at country and global levels. The substantial variation within and between countries suggests it is challenging now to use a single number to represent average immunization costs. This variation can also lead to an open dialogue about ways to reduce variation and improve value for money in immunization services.

\section{Additional file}

Additional file 1: Supplementary material for "The cost determinants of routine infant immunization services: a meta-regression analysis of six country studies". (DOCX $426 \mathrm{~kb})$

\section{Abbreviations \\ DTP3: Third immunization for diphtheria, tetanus, and pertussis; \\ EPIC: Expanded Program on Immunization Costing and Financing; GDP: Gross domestic product; NGO: Non-governmental organization; WAIC: Watanabe-Akaike information criterion}

\section{Acknowledgements}

The authors are thankful for technical input and contextual expertise from Mead Over, Robert Steinglass, Melanie Bertram, Vivian Valdmanis, and Santiago Cornejo (EPIC-2 expert advisory panel members), as well as Sarah Pallas, Jim Hammitt, Sebastien Haneuse, Claudia Rivera, Carol Levin, Stéphane Verguet, Wu Zeng, and Dejan Loncar (writing workshop participants). The authors also thank EPIC-1 study team members and dissemination workshop participants for input on data collection and country-specific analyses and feedback on draft analyses (contributors listed at www.immunizationeconomics.org).

\section{Funding}

This study was funded by the Bill \& Melinda Gates Foundation. LB is employed by the funder (Bill \& Melinda Gates Foundation) and participated in the study design, interpretation of data, and manuscript writing. NAM (corresponding author) had full access to all data and had final responsibility for the decision to submit for publication.

\section{Availability of data and materials}

The datasets supporting the conclusions of this article are available in the Dataverse repository, https://dataverse.harvard.edu/dataverse/EPIC2.

\section{Authors' contributions}

This study was conceived by NAM, CS, LB, and SCR. CS led the creation of the study dataset. ZJW and FG contributed to data preparation, and ZJW created additional variables from Demographic and Health Services (DHS) data. NAM conducted the analysis and developed the first draft of the manuscript. NAM, CS, FG, ZJW, LB, and SCR contributed to interpretation of analytic results and edited the manuscript. All authors read and approved the final manuscript. 


\section{Ethics approval and consent to participate}

Not applicable (secondary analysis of existing data)

\section{Consent for publication}

Not applicable.

\section{Competing interests}

The authors declare that they have no competing interests.

\section{Publisher's Note}

Springer Nature remains neutral with regard to jurisdictional claims in published maps and institutional affiliations.

\section{Author details}

${ }^{1}$ Department of Global Health and Population, Harvard T.H. Chan School of Public Health, Boston, Massachusetts, USA. ${ }^{2}$ Center for Health Decision Science, Harvard T.H. Chan School of Public Health, Boston, Massachusetts, USA. ${ }^{3}$ Bill \& Melinda Gates Foundation, Seattle, Washington, USA.

\section{Received: 31 March 2017 Accepted: 11 September 2017}

\section{Published online: 06 October 2017}

\section{References}

1. Bishai D, McQuestion M, Chaudhry R, Wigton A. The costs of scaling up vaccination in the world's poorest countries. Health Affair. 2006;25(2): 348-56.

2. Wolfson LJ, Gasse F, Lee-Martin SP, Lydon P, Magan A, Tibouti A, Johns B, Hutubessy R, Salama P, Okwo-Bele JM. Estimating the costs of achieving the WHO-UNICEF Global Immunization Vision and Strategy, 2006-2015. Bull World Health Organ. 2008:86(1):27-39.

3. Brenzel L. What have we learned on costs and financing of routine immunization from the comprehensive multi-year plans in GAVI eligible countries? Vaccine. 2015:33 Suppl 1:A93-98.

4. Robertson RL, Davis JH, Jobe K. Service volume and other factors affecting the costs of immunizations in the Gambia. Bull World Health Organ. 1984; 62(5):729-36.

5. Walker D, Mosqueira NR, Penny ME, Lanata CF, Clark AD, Sanderson CF, FoxRushby JA. Variation in the costs of delivering routine immunization services in Peru. Bull World Health Organ. 2004:82(9):676-82.

6. Creese AL, Sriyabbaya N, Casabal G, Wiseso G. Cost-effectiveness appraisal of immunization programmes. Bull World Health Organ. 1982; 60(4):621-32.

7. Brenzel L, Young D, Walker DG. Costs and financing of routine immunization: approach and selected findings of a multi-country study (EPIC). Vaccine. 2015;33 Suppl 1:A13-20.

8. Schutte C, Chansa C, Marinda E, Guthrie TA, Banda S, Nombewu Z, Motlogelwa K, Lervik M, Brenzel L, Kinghorn A. Cost analysis of routine immunisation in Zambia. Vaccine. 2015:33 Suppl 1:A47-52.

9. Janusz CB, Castaneda-Orjuela C, Molina Aguilera IB, Felix Garcia AG, Mendoza L, Diaz IY, Resch SC. Examining the cost of delivering routine immunization in Honduras. Vaccine. 2015;33 Suppl 1:A53-59.

10. Goguadze K, Chikovani I, Gaberi C, Maceira D, Uchaneishvili M, Chkhaidze N, Gotsadze G. Costs of routine immunization services in Moldova: findings of a facility-based costing study. Vaccine. 2015;33 Suppl 1:A60-65.

11. Le Gargasson JB, Nyonator FK, Adibo M, Gessner BD, Colombini A. Costs of routine immunization and the introduction of new and underutilized vaccines in Ghana. Vaccine. 2015;33 Suppl 1:A40-46.

12. Ahanhanzo CD, Huang XX, Le Gargasson JB, Sossou J, Nyonator $F$ Colombini A, Gessner BD. Determinants of routine immunization costing in Benin and Ghana in 2011. Vaccine. 2015;33 Suppl 1:A66-71.

13. Maceira D, Goguadze K, Gotsadze G. The drivers of facility-based immunization performance and costs. An application to Moldova. Vaccine. 2015;33 Suppl 1:A72-78

14. Valdes W, Janusz CB, Molina Aguilera IB, Mendoza L, Diaz IY, Resch S. Tracking financial flows for immunization in Honduras. Vaccine. 2015;33 Suppl 1:A85-92.

15. Guthrie T, Zikusooka C, Kwesiga B, Abewe C, Lagony S, Schutte C, Marinda E, Humphreys K, Motlogelwa K, Nombewu ZC, et al. Mapping financial flows for immunisation in Uganda 2009/10 and 2010/11: new insights for methodologies and policy. Vaccine. 2015;33 Suppl 1:A79-84.
16. WHO Global Health Observatory. Diphtheria tetanus toxoid and pertussis (DTP3) data by country: Global Health Observatory Data Repository. World Health Organisation; 2015

17. World Bank: World Development Indicators: GDP per capita. World Bank; 2016. (http://data.worldbank.org/indicator/NY.GDP.PCAP.CD). Accessed 1 Feb 2016.

18. Brenzel L. Common approach for the costing and financing of routine immunization and new vaccines (EPIC), working paper. Washington DC: Bill \& Melinda Gates Foundation; 2014

19. Center for Health Decision Sciences. EPI Costing and Financing Study Phase 2 (EPIC2) Dataverse. Boston, MA: Center for Health Decision Sciences, Harvard T.H. Chan School of Public Health; 2015. https://dataverse.harvard. edu/dataverse/EPIC2

20. Weinstein MC, Siegel JE, Gold MR, Kamlet MS, Russell LB. Recommendations of the Panel on Cost-Effectiveness in Health and Medicine. JAMA. 1996; 276(15):1253-8

21. Hoffman MD, Gelman A. The No-U-Turn sampler: adaptively setting path lengths in Hamiltonian Monte Carlo. J Mach Learn Res. 2012;15(1):1593-623.

22. Stan Development Team. Stan: a C++ library for probability and sampling, version 2.8.0. URL. 2015. http://mc-stan.org/.

23. R Core Team. R: a language and environment for statistical computing. Vienna, Austria: R Foundation for Statistical Computing: 2013.

24. Gelman A, Hwang J, Vehtari A. Understanding predictive information criteria for Bayesian models. Stat Comput. 2014:24:997-1016.

25. Vehtari A, Gelman A. WAIC and cross-validation in Stan. Helsinki: Aalto University; 2014.

26. Duan N. Smearing estimate: a nonparametric retransformation method. JASA. 1983:78:605-10.

27. O'Hagan A. On outliers rejection phenomena in Bayes inference. J R Stat Soc B. 1979:41:358-67.

28. Manning WG, Mullahy J. Estimating log models: to transform or not to transform? J Health Econ. 2001;20(4):461-94.

29. Brenzel L, Claquin P. Immunization programs and their costs. Soc Sci Med. 1994;39(4):527-36

\section{Submit your next manuscript to BioMed Central and we will help you at every step:}

- We accept pre-submission inquiries

- Our selector tool helps you to find the most relevant journal

- We provide round the clock customer support

- Convenient online submission

- Thorough peer review

- Inclusion in PubMed and all major indexing services

- Maximum visibility for your research

Submit your manuscript at www.biomedcentral.com/submit
BioMed Central 